\title{
BMJ Open Prediction of adverse motor outcome for neonates with punctate white matter lesions by MRI images using radiomics strategy: protocol for a prospective cohort multicentre study
}

Miaomiao Wang, ${ }^{1}$ Heng Liu, ${ }^{1,2}$ Congcong Liu, ${ }^{1}$ Xianjun Li, ${ }^{1}$ Chao Jin, ${ }^{1}$ Qinli Sun, ${ }^{1,2}$ Zhe Liu, ${ }^{1,2}$ Jie Zheng, ${ }^{3}$ Jian Yang ${ }^{1,2}$

To cite: Wang M, Liu H, Liu C, et al. Prediction of adverse motor outcome for neonates with punctate white matter lesions by MRI images using radiomics strategy: protocol for a prospective cohort multicentre study. BMJ Open 2019;9:e023157. doi:10.1136/ bmjopen-2018-023157

\section{- Prepublication history for} this paper is available online. To view these files, please visit the journal online (http://dx.doi. org/10.1136/bmjopen-2018023157).

MW and $\mathrm{HL}$ contributed equally.

Received 2 April 2018

Revised 11 February 2019

Accepted 15 February 2019

\section{Check for updates}

(C) Author(s) (or their employer(s)) 2019. Re-use permitted under CC BY-NC. No commercial re-use. See rights and permissions. Published by BMJ.

${ }^{1}$ Department of Diagnostic Radiology, The First Affiliated Hospital of Xi'an Jiaotong University, Xi'an, China

${ }^{2}$ Department of Biomedical Engineering, School of Life Science and Technology, Xi'an Jiaotong University, Xi'an, China ${ }^{3}$ Clinical Research Centre, The First Affiliated Hospital of Xi'an Jiaotong University, Xi'an, China

Correspondence to

Dr Jian Yang;

yj1118@mail.xjtu.edu.cn

\section{ABSTRACT}

Introduction Punctate white matter lesions (PWML) are prevalent white matter disease in preterm neonates, and may cause motor disorders and even cerebral palsy. However, precise individual-based diagnosis of lesions that result in an adverse motor outcome remains unclear, and an effective method is urgently needed to guide clinical diagnosis and treatment. Advanced radiomics for multiple modalities data can provide a possible look for biomarkers and determine prognosis quantitatively. The study aims to develop and validate a model for prediction of adverse motor outcomes at a corrected age (CA) of 24 months in neonates with PWML.

Methods and analysis A prospective cohort multicentre study will be conducted in 11 Chinese hospitals. A total of 394 neonates with PWML confirmed by MRI will undergo a clinical assessment (modified Neonatal Behavioural Assessment Scale). At a CA of 18 months, the motor function will be assessed by Bayley Scales of Infant and Toddler Development-III (Bayley-III). Mild-to-severe motor impairments will be confirmed using the Bayley-III and Gross Motor Function Classification System at a CA of 24 months. During the data collection, the perinatal and clinical information will also be recorded. According to the radiomics strategy, the extracted imaging features and clinical information will be combined for exploratory analysis. After using multiple-modelling methodology, the accuracy, sensitivity and specificity will be computed Internal and external validations will be used to evaluate the performance of the radiomics model.

Ethics and dissemination This study has been approved by the institutional review board of The First Affiliated Hospital of Xi'an Jiaotong University (XJTU1AF2015LSK-172). All parents of eligible participants will be provided with a detailed explanation of the study and written consent will be obtained. The results of this study will be published in peer-reviewed journals and presented at local, national and international conferences. Trial registration number NCT02637817; Pre-results.

\section{INTRODUCTION}

Punctate white matter lesions (PWML) are common in neonates and have been found in
Strengths and limitations of this study

- This study will provide an individual-based prediction model of motor outcome in neonates with punctate white matter lesions (PWML), which could contribute to clinical decision-making and selection of the appropriate time for intervention.

- An automatic detection and segmentation pipeline for PWML will be developed for rapid and accurate identification of responsible lesions.

- In order to ensure the sensitivity, specificity and accuracy of the prediction model, other PWML-related impairments will not be considered.

more than $20 \%$ of preterm neonates. ${ }^{1}$ These lesions can be detected on conventional magnetic resonance images and are defined as hyperintensity on $\mathrm{T} 1$ weighted imaging (T1WI) and hypointenity on T2 weighted imaging (T2WI). ${ }^{1-3}$ PWML influence brain development, lead to changes in white matter microstructures and are generally associated with motor deficits and even cerebral palsy (CP), ${ }^{1245}$ although impairments in cognition and vision have also been demonstrated in some subjects. ${ }^{6-9}$ Early accurate identification of a lesion causing adverse motor development is crucial for appropriate counselling of parents of babies, as well for better decision-making regarding interventional strategies in these babies to improve outcomes.

MRI has been widely used for diagnosis, prognosis, treatment monitoring and research in various paediatric neurological diseases. It appears to be considerably sensitive in demonstrating the existence and extent of PWML in neonates. ${ }^{10}$ Conventional MRI (T1WI and T2WI) had been used to provide a prior knowledge that there was a possible relationship between the number and location 
of PWML and adverse motor outcomes. ${ }^{11-13}$ PWML are associated with more widespread abnormalities than those observed on conventional MRI images. ${ }^{31014}$ Further diffusion tensor imaging (DTI) studies showed that white matter damage in the lesions is associated with increased radical diffusivity (RD), decreased fractional anisotropy (FA) and higher lesion load in the corticospinal tract may preliminarily predict later motor impairment. ${ }^{3} 1012$ However, PWML are distributed variably in location and number, and may easily accompany other abnormalities, an early quantitative and individualised prediction system for use in the clinical field is still lacking.

To this end, radiomics is an emerging approach, that has provided insights into several areas of medicine, particularly in clinical oncology. ${ }^{15}$ The main process of radiomics analysis includes high-throughput extraction of quantitative features that result in the conversion of medical images into mineable data and detection of crucial characteristics for supporting decision-making. ${ }^{16}$ Therefore, radiomics combined with clinical information could provide a better understanding of the disease and hopefully improve the accuracy of prediction of the motor disability in neonates with PWML.

\section{Aims}

Primary aim

The aim of this prospective cohort is to develop and validate a model for prediction of adverse motor outcomes (motor delay) in neonates with PWML.

\section{Secondary aims}

1. To establish a practical method for automatic detection and segmentation of PWML.

2. To identify differences in structural MRI characteristics (cortical/deep grey matter and white matter) between neonates with PWML in the neonatal period, who do and do not progress to display motor disability at a corrected age (CA) of 24 months.

3. To investigate the DTI network properties between PWML neonates with and without motor disability.

\section{METHODS AND ANALYSIS}

\section{Study design}

A multicentre prospective cohort study will be conducted among neonates with PWML.

\section{Study setting}

The study will be performed at The First Affiliated Hospital of Xi'an Jiaotong University, Shenzhen Children's Hospital, General Hospital of Ningxia Medical University, Xijing Hospital of the Fourth Military Medical University, Shengjing Hospital of China Medical University, The Second Affiliated Hospital of Wenzhou Medical University, Affiliated Hospital of Zunyi Medical University, Shaanxi Provincial People's Hospital, Baoji Central Hospital, Children's Hospital of Fudan University and Hainan General Hospital, China. It will be begin in June 2018 and expected to be complete in December 2021.

\section{Study sample and recruitment}

Study participants

Previous studies and prior knowledge suggested that the main high-risk factors for PWML include greater birth weight, grade II to III intraventricular haemorrhage, ${ }^{4}$ congenital heart disease, ${ }^{17}$ hypoxic-ischaemic encephalopathy, hypoglycaemia, respiratory infection, septicaemia, hyperbilirubinemia, placenta previa, premature rupture of membranes and amniotic fluid pollution. In general, neonates with one or more of these high-risk factors admitted to the above-mentioned 11 hospitals will be recruited and screened for MRI.

\section{Inclusion criteria}

Neonates who meet all of the following criteria will be considered for enrollment into this study:

1. Neonates with high-risk factors related to PWML;

2. Neonates require MRI clinically to exclude or assess brain injury, or newborn parents who are willing to participant in this study;

3. PWML diagnosed by conventional MRI (T1WI and T2WI);

4. Gestation age (GA) $<42$ weeks and postnatal age at MRI examination $\leq 28$ days;

5. Complete imaging data (T1WI, T2WI and DTI).

\section{Exclusion criteria}

Neonates who meet any of the following criteria will be excluded from this study:

1. Congenital malformation;

2. Genetic metabolic disorders;

3. Obvious image artefacts.

\section{Withdrawal criteria}

Subjects will be withdrawn from the study if any of the following conditions occurs:

1. Complications affecting safety and the outcome of illness (such as trauma and tumour);

2. Inability to complete follow-up examination.

\section{Sample size calculations}

There are no directly available data to assess the relationship between MRI and clinical assessments of PWML neonates to predict motor developmental outcomes or even CP at a CA of 24 months. Sample size calculations are based on a similar previous study, that investigated the ability of MRI at term-equivalent age (TEA) to evaluate neurodevelopmental outcomes (especially for motor development) at 24 months of age. ${ }^{4}$ In the prospective study of preterm neonates with PWML at TEA in a total sample size of $n=29$, the numbers of infants with motor abnormality was nine $(31.0 \%) .{ }^{4}$ We assume that the same incidence rate of adverse motor outcomes in PWML neonates will be observed in our study. In addition, the incidence of motor delay in the subjects without PWML is assumed to be $3 \% .{ }^{18}$ Previous study reported that the lesion volume is considered to be an important indicator of motor prognosis. ${ }^{13}$ According to our 
preliminary experiments, when the lesion volume is set as the most important variable, the R-square to other covariates (the birth history [GA, delivery mode, gender, BW, head circumference, body length, mNBAS scores, Apgar scores and age at MRI], whole brain volume and socioeconomic status variables) is calculated to be 0.56 . If $\alpha=0.05,1-\beta=0.95$, then the study requires 82 PWML neonates to be enrolled using G*Power V.3.1.9.2. ${ }^{19}{ }^{20}$ After review of similar literatures, we plan to enrol 328 PWML neonates (four times of the calculated sample size) to meet the requirements of training, validation and testing of the radiomics model. Based on previous follow-up experience and given an anticipated dropout rate of $20 \%$, a total sample size of 394 neonates with PWML will be needed; of these, $315(80 \%)$ random subjects will be assigned to training and validation datasets and the remaining $79(20 \%)$ subjects will be classified as the test dataset.

\section{Perinatal and clinical data collection}

The details of the pregnancy, birth history and neonatal clinical diagnosis and socioeconomic status will be collected from the medical records. This will allow identification of risk factors for motor impairments, and help to establish predictive variables.

A number of prenatal factors have been shown to be associated with brain injury and have an effect on shortterm and long-term outcomes. Premature rupture of membranes (PROM), most commonly defined as rupture of membranes before the onset of labour, increases the risk of infection-associated morbidity to the fetus and has a major impact on outcomes. ${ }^{21}$ Compared with singletons, multiple birth status increase the risk of

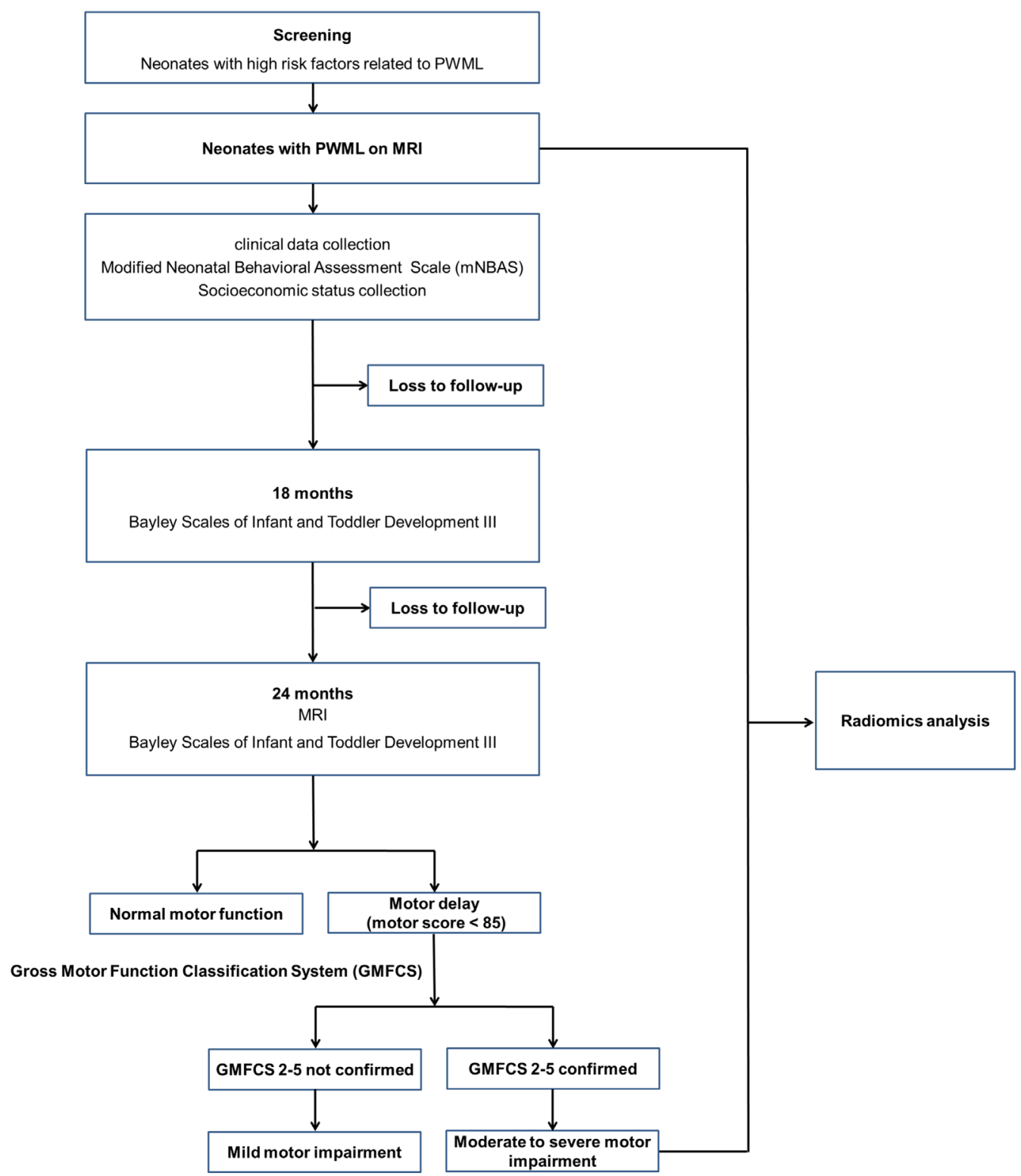

Figure 1 Flow chart of the study protocol. PWML, punctate white matter lesions. 
CP. ${ }^{22}{ }^{23}$ Furthermore, a systematic review reported significant associations of placental abnormalities and emergency caesarean section and CP. ${ }^{24}$ Exposure to maternal pre-eclampsia and intrauterine growth restriction seem to have a negative impact on cognitive function in school-aged children. ${ }^{25}$ Maternal infection, parental age and a smoking history are predominantly related to the impaired behavioural function. ${ }^{26}$

Birth history variables collected will include GA at birth, delivery mode, gender, birth weight (BW), head circumference, body length, Apgar score, age at MRI scan and neurodevelopmental assessment scale (modified Neonatal Behavioural Assessment Scale [mNBAS]) score. Several studies have demonstrated that the likelihood of adverse neurodevelopmental outcomes increase with decreasing GA, lower BW and lower Apgar scores, and multiple studies have reported poorer outcomes in male neonates. ${ }^{23}{ }^{27-29}$ In addition, these variables can be used to adjust for confounders between groups.

Clinical information will be gathered for each neonate from birth until discharge from hospital. Socioeconomic status such as parental education and occupation, family structure and family income will be collected using a parent questionnaire. During the follow-up, the treatment history will be recorded in detail based on the medical records.

\section{Study procedures}

The study procedures are shown in figure 1. Subjects will be enrolled through inclusion and exclusion criteria described above. When the neonates with high-risk factors of PWML are in a medically stable condition, they will undergo an MRI examination. There is no established gold standard neurological assessment for neonates, so the mNBAS will be used within the 7 days of the MRI. Neurobehavioral development will be assessed by a designated experienced neonatologist blinded to the MRI findings and any unrelated medical information (such as babies' clinical condition and other examination results irrelevant to the content of the current assessment) at each site.

At a CA of 18 months (range;+/-2 weeks), the families will be invited back to the birth hospital for the first follow-up assessment of their child's neurodevelopmental outcome. Experienced paediatricians blinded to the medical history will assess each infant's neurodevelopment using the Bayley Scales of Infant and Toddler Development-III (Bayley-III). The infants and their parents will be contacted to schedule the second visit for an MRI examination at a CA of 24 months (range;+/-2 weeks). At the same time, Bayley-III and Gross Motor Function Classification System (GMFCS) will be provided to confirm the motor developmental outcomes. The adverse motor outcome is first classified by a motor score $<85$ (Bayley-III). Next, the GMFCS will be measured in the infants with motor score $<85$. The subjects confirmed to have a GMFCS of 2 to 5 will be defined as having moderate-to-severe motor impairment, and others who do not
Table 1 The three-dimensional T1 weighted imaging parameters for different scanners

\begin{tabular}{llll}
\hline & GE & Philips & Siemens \\
\hline Repetition time $(\mathrm{ms})$ & 10.18 & 7.57 & 1900 \\
Echo time $(\mathrm{ms})$ & 4.62 & 4.60 & 3.37 \\
Slice thickness $(\mathrm{mm})$ & 1 & 1 & 1 \\
Gap $(\mathrm{mm})$ & 0 & 0 & 0 \\
Field of view $\left(\mathrm{mm}^{2}\right)$ & $256 \times 256$ & $256 \times 256$ & $256 \times 256$ \\
Matrix size & $256 \times 256$ & $256 \times 256$ & $256 \times 256$ \\
\hline
\end{tabular}

have a GMFCS of 2 to 5 will be defined as having mild motor impairment. Considering the developmental differences of preterm and term neonates, the collected data will first be analysed separately.

The questionnaire data will be collected by trained interviewers during a telephone survey for guardians who are reluctant to come to the hospital for follow-up. During the study, if any of the protocols (eg, the MRI sequence parameters) need to be modified because of the circumstances at particular centres, an application should be submitted to the ethics committee for review.

\section{ASSESSMENT PROCEDURE \\ MRI methods}

Image acquisition

Brain MRI will be performed using 3.0T scanners with 8-channel head coils. Noise from MRI will be attenuated using micro-earplugs. In order to reduce motion artefacts and complete the MRI examination, all subjects will be required to scan under natural sleep after adjusting their sleep and feed times. If necessary (subjects unable to remain for the MRI examination), sedation will be taken after obtaining parental consent. The potential risks of sedatives will be fully considered, and the subjects will be monitored for 24 hours to see if there is an adverse drug reaction. Each subject's head will be immobilised by moulded foam, which is placed around the head. The MRI sequences will include T1WI, T2WI and DTI, and the total scan time will be approximately $20 \mathrm{~min}$. When the motion artefacts of the conventional MRI images (T1WI and T2WI) are significant

\begin{tabular}{llll}
\hline $\begin{array}{l}\text { Table } 2 \\
\text { scanners }\end{array}$ & The T2 weighted imaging parameters for different \\
& GE & Philips & Siemens \\
\hline Repetition time $(\mathrm{ms})$ & 2000 & 2500 & 2000 \\
Echo time $(\mathrm{ms})$ & 73.11 & 253.99 & 83 \\
Slice thickness $(\mathrm{mm})$ & 1 & 1 & 1 \\
Gap $(\mathrm{mm})$ & 0 & 0 & 0 \\
Field of view $\left(\mathrm{mm}^{2}\right)$ & $180 \times 180$ & $180 \times 180$ & $180 \times 180$ \\
& $(240 \times 240)$ & $(240 \times 240)$ & $(240 \times 240)$ \\
Matrix size & $180 \times 180$ & $180 \times 180$ & $180 \times 180$ \\
& $(240 \times 240)$ & $(240 \times 240)$ & $(240 \times 240)$ \\
\hline
\end{tabular}




\begin{tabular}{llll}
$\begin{array}{l}\text { Table } 3 \text { The diffusion tensor imaging } \\
\text { different scanners }\end{array}$ & parameters for \\
\hline & 11000 & 9000 & 8800 \\
\hline Repetition time $(\mathrm{ms})$ & 69.5 & 91 & 57 \\
\hline Echo time $(\mathrm{ms})$ & 0,1000 & 0,1000 & 0,1000 \\
b values $\left(\mathrm{s} / \mathrm{mm}^{2}\right)$ & 32 & 32 & 32 \\
Numbers of diffusion & & & \\
gradient directions & 1 & 1 & 1 \\
Number of excitation & 2.0 & 2.0 & 2.0 \\
Slice thickness $(\mathrm{mm})$ & 0 & 0 & 0 \\
Gap (mm) & $180 \times 180$ & $180 \times 180$ & $180 \times 180$ \\
Field of view $\left(\mathrm{mm}{ }^{2}\right)$ & $(240 \times 240)$ & $(240 \times 240)$ & $(240 \times 240)$ \\
& $90 \times 90$ & $90 \times 90$ & $90 \times 90$ \\
Matrix size & $(120 \times 120)$ & $(120 \times 120)$ & $(120 \times 120)$ \\
& & &
\end{tabular}

to affect the diagnosis, the sequence will be repeated. While for DTI, whether images with moderate-to-severe motion artefacts will be scanned repeatedly depends on the state of the baby. Providing that the subjects are well-prepared before examination, the probability of neonates completing the three successful sequences is close to $90 \%$ at our hospital. Therefore, the setting of the protocols is suitable for promotion at other sites. The details of the protocols used at the different sites are shown in tables 1-3. Vital signs will be monitored during the MRI examination. If an adverse event occurs, the scan will be stopped immediately and professionally handled depending on the situation.

\section{Image analysis}

MRI data will be analysed by using image processing techniques as follows:

\section{Structural data analysis}

The follow-up T1WI data will be segmented using SPM V.12.0.$^{3031}$ The grey matter volume, white matter volume and total brain volume will be calculated using the native space probabilistic tissue maps produced during segmentation. Statistical analysis will use voxel-based analysis techniques.

\section{Diffusion data analysis}

Preprocessing and quality control will be used to detect and correct motion artefacts and image distortions. This adopted workflow will consist of brain extraction, rigid registration, distortion correction, artefacts rejection and spatial smoothing. Rigid registration will be utilised to correct misalignments. Motion artefacts will be rejected by using local Pearson correlation coefficient. ${ }^{32}$ FA, RD, axial diffusivity $(\mathrm{AD})$ and mean diffusivity (MD) will be estimated from preprocessed diffusion data using a diffusion tensor model. Whole brain voxel-based analysis of diffusion parameters will be performed using optimised tract-based spatial statistics. $^{33}$ Probabilistic tractography will be performed using automated fiber-tract quantification. ${ }^{34}$ Furthermore, the brain structural network based on the diffusion data will be constructed including cortical parcellation, tractography and generation of a connectivity matrix. ${ }^{35}$

\section{Harmonisation analysis}

Images collected from multiple sites are prone to technical variability across scans, including heterogeneity in the imaging protocol, variations in the scanning parameters and differences between the manufacturers of the scanners. ${ }^{36}$ In order to remove the bias and variability caused by the unwanted site effects, the ComBat model will be used for harmonisation of data from different scanners and centres. This method adjusts the mean value and variance of different groups by combining the location/scale model and empirical Bayes framework. ${ }^{3738}$ Fortin et al have demonstrated that the ComBat method could be better for harmonisation of DTI and cortical thickness measurement, which not only preserves biological variability but also removes the unwanted variation introduced by site. ${ }^{37} 39$

\section{Workflow of radiomics analysis}

First, the MRI images acquired from each subject will be preprocessed to ensure that they are consistent. Image contrast enhancement and removal of noise and artefacts will also be needed. The PWML will then be segmented. Segmentation of the lesions will be performed from manual to semi-automated and fully automated by applying the deep convolutional neural network. Second, features of the lesions will be extracted, and useful information selected to assist in the characterisation of PWML neonates with different motor developmental outcomes. The features are mainly comprised of spatial relationships, shapes and sizes, textural features and the filtered-based information of the lesions. Third, the useful radiomic features selected from the poll of extracted features will be combined with clinical information for exploratory analysis. During this process, groups of highly correlated imaging features can be identified via clustering, and these features can be reduced to single archetypal features per cluster. Furthermore, features that have well-correlated clinical indexes will be excluded because they will not provide additional information. In the final stage, radiomic modelling includes three main aspects, that is, feature selection, modelling methodology and validation. In order to avoid overfitting, features lacking robustness against sources of variability will be further eliminated. Multiple-modelling methods will then be used to choose the optimal one for this target PWML population. Validation techniques are critical tools for evaluation of the performance of a model, so internal and external validations will be performed in our study. ${ }^{1640} 41$ The radiomics processing workflow is shown in figure 2 .

\section{Clinical assessments}

Modified neonatal behavioural assessment scale

The Neonatal Behavioural Assessment Scale (NBAS) is a neurobehavioral scale designed to examine a neonate's responses to a new environment, such as the ability to orient and habituate to visual or auditory stimuli, both animate and inanimate; the quality of motor tone 


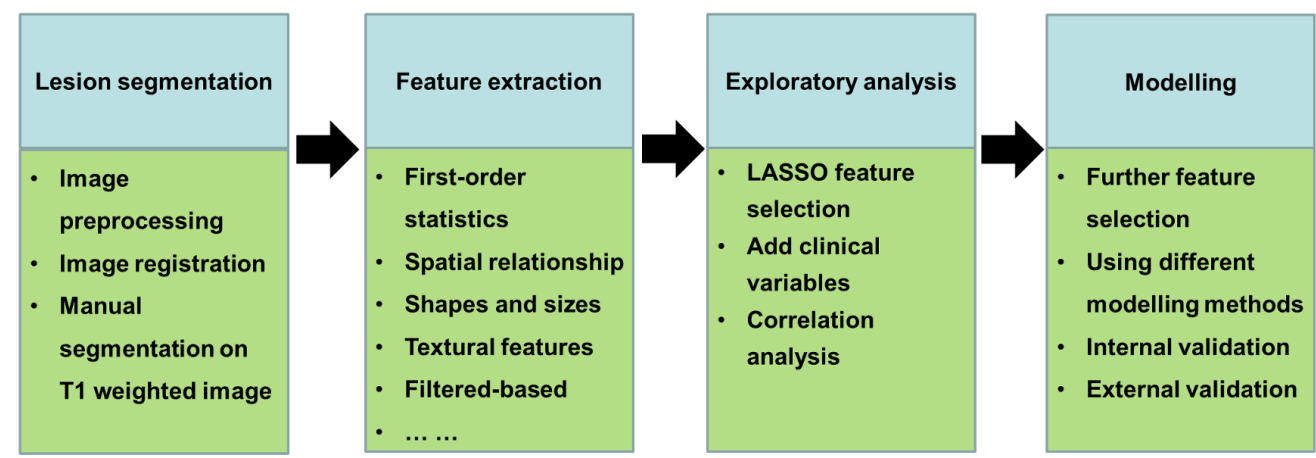

Figure 2 The workflow of radiomic analysis. LASSO, least absoluted shrinkage and selection operator.

and activity levels and the infant's level of state regulation. ${ }^{42}$ The NBAS contains 28 behavioural items scored for optimal performance, 18 reflex items and nine supplementary items to assess the general aspects of overall infant behaviour. ${ }^{43}$ It has been demonstrated to contribute to identification of newborn behaviour and development affected by prenatal and perinatal variables, such as intrauterine growth restriction, intrauterine drug exposure, low BW and prematurity. ${ }^{44}$ Additionally, the NBAS is beneficial in terms of early interventions and to help predict outcome. ${ }^{45}$ The mNBAS (20 items) is established by combining the advantages of NBAS and a large number of clinical experiences based on the China's national conditions. This method is practical, reliable and more suitable for Chinese neonates. ${ }^{46}$ Furthermore, it takes only $10 \mathrm{~min}$ to complete the evaluation of this scale. The predictive validity of the mNBAS has been established in babies with asphyxia. The sensitivity and specificity were $88.9 \%$ and $82.6 \%$, respectively, at 7 days after birth and $84.6 \%$ and $97.4 \%$ respectively, at 12 to 14 days. ${ }^{46}$ Furthermore, several studies confirmed the validity of the scale. ${ }^{4748}$

\section{Bayley scales of infant and toddler development-III}

The Bayley-III is a validated and widely-used neurodevelopmental screening instrument designed to measure cognitive, language (receptive and expressive communication) and motor (fine and gross motor) development in children ages 1 to 42 months, and takes 40 to $60 \mathrm{~min}$ to perform for a cooperative baby. The fine motor subtest provides an assessment of eye movements, perceptual-motor integration, motor planning and motor speed. The gross motor subtest measures movements of the limbs and torso. ${ }^{49}$ Normed scores on this scale have a mean of 100 and a SD of 15 , where higher scores reflect a better development. Several studies have been performed and demonstrated the high acceptability and reliability (0.95 0.99) for the cognitive and motor subscales. ${ }^{50} 51$ The motor scores are transformed into the following classifications: scores $<85$ indicating 'motor delay'; scores 85 to 114 indicating 'within normal limits'; scores $\geq 115$ indicating 'accelerated motor performance'. ${ }^{52}$ In this study, infants with normal and accelerated motor performance will be classified as having normal motor function.

\section{Gross motor function classification system}

The GMFCS is developed to classify the gross motor function of children with $\mathrm{CP}^{53}$ The GMFCS is divided into five levels, and as the level increases the motor function is worse. Classification of motor abilities is dependent on age, and the GMFCS has separate descriptions for several age bands for each level: before the age of 2 years, from the age of 2 to 4 years, from 4 to 6 years and from 6 to 12 years, with an adolescent age band now available. Studies have reported high inter-rater reliability $(0.93)$, test-retest reliability $(0.79)$ and stability of the first and last ratings (weighted kappa coefficient, 0.84). ${ }^{54} 55$ Adverse motor outcomes are further divided into mild and moderate-to-severe motor impairment groups according to level 2 to 5 of the GMFCS. ${ }^{12}$

\section{Blinding}

The radiologists involved in the MRI analysis will be blinded to other imaging results, clinical information and assessment findings. The neonatologists carrying out the clinical assessments will be blinded to the MRI findings. Follow-up assessments at a CA of 18 months and 24 months will be scored by paediatricians blinded to the perinatal history, neonatal assessment and MRI findings.

\section{Confidentiality}

The original data will be preserved by The First Affiliated Hospital of Xi'an Jiaotong University, China. Unless required by law, the patient status will not be divulged. Anonymised study data will be published for scientific purpose.

\section{Statistical analysis}

The data analyses will be supervised by an experienced statistician. All measurement variables that are in a normal distribution will be expressed as the mean $\pm \mathrm{SD}$, and categorical variables will be shown as numbers and percentages. Continuous variables will be compared using the independent samples t-test or the Mann-Whitney test, and categorical variables will be compared by using the Chi-square test or the Fisher exact test. Missing data will be handled using full information maximum likelihood in the estimation of path models, which is robust against biases from data missing at random. Models will 
be constructed to identify the risk factors or predict the motor developmental outcome of neonates with PWML, the univariable analysis will be used first to look for variables that are statistically significant $(\mathrm{p}<0.05)$ and these variables will then be selected for multivariable models. Some classifiers (such as support vector machine, logistic regression and k-nearest neighbour) will be used to divide the predictors into categories on the basis of the motor outcome of the neonates, and then the best one will be chosen. During the data analysis, the correlation relationship will be analysed for the selected features and clinical indices (eg, perinatal history, clinical information and socioeconomic status). The discriminatory performance and stability of the model was validated using 10-fold cross-validation in which the original set was randomly partitioned in 10 equal size subsamples. Of the 10 subsamples, a single subsample is retained as the validation set for testing the model, and the remaining nine subsamples are used as the training set. The cross-validation process is repeated 10 times with each of the subsamples used exactly once as the validation set. Moreover, the external validation will be performed using another independent dataset. The area under the receiver operating characteristic curve will be used to evaluate how the model can allow identification of PWML neonates with motor delay. The sensitivity, specificity, positive predictive value, negative predictive value and accuracy will be calculated. A nomogram will be used for visualisation of the optimal prediction model.

\section{Data management}

The original data for the participants will be kept confidential throughout the study. The electronic data will be gathered by a special researcher at each site and supervised by the two researchers at our centre. The data and backup information will be stored on a secure computer file and only study members will have access to the data. In addition, the clinical research centre at our hospital and The Ministry of Science and Technology are responsible for study and data supervision. Through annual reviews, they can obtain interim and final results.

\section{Ethics and information dissemination}

All participating centres have approved the study protocol. Ethical approval for the study has been given by the institutional review board of The First Affiliated Hospital of Xi'an Jiaotong University (XJTU1AF2015LSK-172). The trial has been registered with the Clinical Trials Registry (NCT02637817). All of the parents of eligible participants will be provided with detailed explanation of the study and obtain the written informed consent. Subjects may withdraw from the study at any time without explanation. The results will be published in peer-reviewed journals and presented in local, national and international conferences. The person who has the authorship eligibility is the researcher who contributes to the experiment. Since this project is supported by the national foundation, the access to the full protocol, participant-level dataset and statistical code will be decided by the government.

\section{Patient and public involvement}

No patients or their guardians were involved in setting the research question or the outcome measures nor were they involved in the design or implementation of the study. No guardians of patients were asked to advise on interpretation or writing up of results. Guardian representatives have advised on the dissemination of results, including the use of electronic mails and social media.

\section{DISCUSSION}

The protocol combines brain MRI, clinical information and assessments at the neonatal stage to establish an individual-based model to predict motor disability at a CA of 24 months of neonates with PWML.

There will be some strengths to this study. The advanced radiomics method provides a new insight for clinicians to extract many significant imaging and clinical features to establish an individual prediction model based on motor developmental outcomes. Previous studies of PWML have mostly had retrospective designs, and their sample sizes were relatively small. The researchers mainly focused on the different MRI characteristics between neonates with and without PWML, and the macrostructural features of the lesions. ${ }^{36-58}$ According to prior knowledge, previous studies reported that the location, volume of lesions and some of the diffusion parameters can preliminarily predict the adverse motor or cognitive outcomes, but few of them were longitudinal. ${ }^{2} 1359$ All were based on comparison at the group level and the predictive values need to be further validated. This study will provide an individual-based prediction model of motor outcome via a prospective cohort design, which will may contribute to decision-making and choice of the appropriate time for intervention. Furthermore, the combination of multiple modalities in our study will improve the ability to predict outcomes to a greater extent than a single modality alone.

Our hospital had developed an individualised examination procedure for newborns and infants, and we have already extended it to other participating sites to ensure the feasibility of the process. In addition, the MRI sequences and clinical neurological assessments are the most commonly used clinically. With the proliferation of multi-site neuroimaging studies, there is a great need to handle the non-biological variance introduced by differences in MRI scanners and acquisition protocols. Such unwanted sources of bias and variability can hinder the detection of features associated clinical covariates of interest and cause spurious findings. ${ }^{396-62}$ To remove the site effects in the multi-site studies, the ComBat has been found to be an effective harmonisation technique that both removes unwanted variation associated with the site and preserve biological associations in the data. ${ }^{37}{ }^{39}$ Moreover, we will also try to apply a deep learning algorithm to better deal with this critical issue. Neonatal brains 
differ significantly from those of adults. The immature myelination of the white matter in neonatal brains results in inversion of MRI contrast when compared with adult brain scans. ${ }^{63}$ This requires image processing to be performed on T2WI rather than T1WI. ${ }^{64}$ However, the PWML is mainly detected in T1WI, and this makes the lesion segmentation more challenging. Finally, this study will develop an automatic detection and segmentation pipeline for PWML by multiple repeated training and testing the manual labels on T1WI. It will not only help to identify PWML rapidly and consistently, but it will also provide the foundation for individualised multi-sequence fusion to locate responsible lesions.

We need to point out several limitations of the current study protocol. PWML can be associated with motor, cognitive or vision impairments, but we will only be focusing on adverse motor developmental function to improve the sensitivity, specificity and accuracy of the prediction model in a specific aspect. Other functional deficits will be analysed further based on the pipeline developed in this protocol. Neuroimaging with functional MRI has great potential as a way of characterising typical development and detecting abnormality early. ${ }^{65}$ However, functional MRI is not included in our study as a necessary sequence because of the tolerance of neonates and infants in the MRI scanning room. On account of this technique being highly sensitive to head motion during scanning, and after the conventional and diffusion sequences, the successful rate is not guaranteed. In addition, the follow-up duration to a CA of 24 months is not long enough. However, independent of this study, we will attempt to follow them up until they are adults.

Acknowledgements We would like to thank Dr Yungeng Gan from Shenzhen Children's Hospital, Dr Yulin Guo from General Hospital of Ningxia Medical University, Dr Hong Yin from Xijing Hospital of the Fourth Military Medical University, Dr Xiaoming Wang from Shengjing Hospital of China Medical University, Dr Zhihan Yan from The Second Affiliated Hospital of Wenzhou Medical University, Dr Tijiang Zhang from Affiliated Hospital of Zunyi Medical University, Dr Xiaoling Zhang from Shaanxi Provincial People's Hospital, Dr Zhuanqin Ren from Baoji Central Hospital, Dr Zhongwei Qiao from Children's Hospital of Fudan University and Dr Feng Chen from Hainan General Hospital, China. We are also grateful to Dr Liang Wu from Shaanxi Kangfu Hospital, Dr Xihui Zhou in the neonatology department and Dr Jie Yue in the pediatric department from The First Affiliated Hospital of Xi'an Jiaotong University. We are thankful to the patients and their guardians who participated in the study.

Contributors All named authors contributed to the design of this trial protocol, to drafting and revising the manuscript and have approved this version for submission. MMW, HL and CCL designed and conceptualised the study, and developed the first version of the manuscript. XJL and CJ provided assistance for data acquisition protocol and radiomics methodology. QLS, ZL and JZ helped to perform statistical analysis consideration and revised manuscript for important intellectual content. JY carried out the design and revised the final version of the manuscript critically.

Funding This work was supported by the grant from National Key Research and Development Program of China (2016YFC0100300), the National Natural Science Foundation of China (No.81771810, 81471631, 51706178), the 2011 New Century Excellent Talent Support Plan of the Ministry of Education, China (NCET-11-0438) and the Clinical Research Award of the First Affiliated Hospital of Xi'an Jiaotong University (XJTU1AF-CRF-2015-004).

Competing interests None declared.

Patient consent for publication Parental/guardian consent obtained.
Ethics approval The First Affiliated Hospital of Xi'an Jiaotong University (XJTU1AF2015LSK-172).

Provenance and peer review Not commissioned; externally peer reviewed.

Data sharing statement Data sharing: No additional data are available.

Open access This is an open access article distributed in accordance with the Creative Commons Attribution Non Commercial (CC BY-NC 4.0) license, which permits others to distribute, remix, adapt, build upon this work non-commercially, and license their derivative works on different terms, provided the original work is properly cited, appropriate credit is given, any changes made indicated, and the use is non-commercial. See: http://creativecommons.org/licenses/by-nc/4.0/.

\section{REFERENCES}

1. Kersbergen KJ, Benders MJ, Groenendaal F, et al. Different patterns of punctate white matter lesions in serially scanned preterm infants. PLoS One 2014;9:e108904.

2. Tortora D, Panara V, Mattei PA, et al. Comparing $3 T$ T1-weighted sequences in identifying hyperintense punctate lesions in preterm neonates. AJNR Am J Neuroradiol 2015;36:581-6.

3. Bassi L, Chew A, Merchant N, et al. Diffusion tensor imaging in preterm infants with punctate white matter lesions. Pediatr Res 2011;69:561-6.

4. de Bruïne FT, van den Berg-Huysmans AA, Leijser LM, et al. Clinical implications of MR imaging findings in the white matter in very preterm infants: a 2-year follow-up study. Radiology 2011;261:899-906.

5. Jeon TY, Kim JH, Yoo SY, et al. Neurodevelopmental outcomes in preterm infants: comparison of infants with and without diffuse excessive high signal intensity on MR images at near-term-equivalent age. Radiology 2012;263:518-26.

6. Back SA, Miller SP. Brain injury in premature neonates: a primary cerebral dysmaturation disorder? Ann Neurol 2014;75:469-86.

7. Nosarti C, Giouroukou E, Micali N, et al. Impaired executive functioning in young adults born very preterm. $J$ Int Neuropsychol Soc 2007;13:571-81.

8. van Tilborg E, Achterberg EJM, van Kammen CM, et al. Combined fetal inflammation and postnatal hypoxia causes myelin deficits and autism-like behavior in a rat model of diffuse white matter injury. Glia 2018;66:78-93

9. Bassi L, Ricci D, Volzone A, et al. Probabilistic diffusion tractography of the optic radiations and visual function in preterm infants at term equivalent age. Brain 2008;131:573-82.

10. Li X, Gao J, Wang M, et al. Characterization of Extensive Microstructural Variations Associated with Punctate White Matter Lesions in Preterm Neonates. AJNR Am J Neuroradiol 2017;38:1228-34.

11. Arberet C, Proisy M, Fausser JL, et al. Isolated neonatal MRI punctate white matter lesions in very preterm neonates and quality of life at school age. J Neonatal Perinatal Med 2017:10:257-66.

12. Tusor N, Benders MJ, Counsell SJ, et al. Punctate White Matter Lesions Associated With Altered Brain Development And Adverse Motor Outcome In Preterm Infants. Sci Rep 2017;7:13250.

13. Guo T, Duerden EG, Adams E, et al. Quantitative assessment of white matter injury in preterm neonates: Association with outcomes. Neurology 2017;88:614-22.

14. Chau V, Synnes A, Grunau RE, et al. Abnormal brain maturation in preterm neonates associated with adverse developmental outcomes. Neurology 2013;81:2082-9.

15. Huang Y, Liu Z, He L, et al. Radiomics signature: a potential biomarker for the prediction of disease-free survival in early-stage (I or II) Non-Small Cell Lung Cancer. Radiology 2016;281:947-57.

16. Gillies RJ, Kinahan PE, Hricak H. Radiomics: images are more than pictures, they are data. Radiology 2016;278:563-77.

17. Guo T, Chau V, Peyvandi S, et al. White matter injury in term neonates with congenital heart diseases: Topology \& comparison with preterm newborns. Neuroimage 2019;185:742-9.

18. Shevell M, Ashwal S, Donley D, et al. Practice parameter: evaluation of the child with global developmental delay: report of the Quality Standards Subcommittee of the American Academy of Neurology and The Practice Committee of the Child Neurology Society. Neurology 2003;60:367-80.

19. Faul F, Erdfelder E, Lang AG, et al. G*Power 3: a flexible statistical power analysis program for the social, behavioral, and biomedical sciences. Behav Res Methods 2007;39:175-91.

20. Faul F, Erdfelder E, Buchner A, et al. Statistical power analyses using $\mathrm{G}^{*}$ Power 3.1: tests for correlation and regression analyses. Behav Res Methods 2009;41:1149-60. 
21. Xia H, Li X, Li X, et al. The clinical management and outcome of term premature rupture of membrane in East China: results from a retrospective multicenter study. Int J Clin Exp Med 2015;8:6212-7.

22. Gnanendran L, Bajuk B, Oei J, et al. NICUS Network. Neurodevelopmental outcomes of preterm singletons, twins and higher-order gestations: a population-based cohort study. Arch Dis Child Fetal Neonatal Ed 2015;100:F106-14.

23. Colver A, Fairhurst C, Pharoah POD. Cerebral palsy. The Lancet 2014;383:1240-9.

24. Mclntyre S, Taitz D, Keogh J, et al. A systematic review of risk factors for cerebral palsy in children born at term in developed countries. Dev Med Child Neurol 2013;55:499-508.

25. Morsing E, Maršál K. Pre-eclampsia- an additional risk factor for cognitive impairment at school age after intrauterine growth restriction and very preterm birth. Early Hum Dev 2014;90:99-101.

26. Oerlemans AM, Burmanje MJ, Franke B, et al. Identifying Unique Versus Shared Pre- and Perinatal Risk Factors for ASD and ADHD Using a Simplex-Multiplex Stratification. J Abnorm Child Psychol 2016;44:923-35.

27. Nelson KB, Blair E. Prenatal factors in singletons with cerebral palsy born at or near term. N Engl J Med 2015;373:946-53.

28. Linsell L, Malouf R, Morris J, et al. Prognostic factors for poor cognitive development in children born very preterm or with very low birth weight. JAMA Pediatr 2015;169:1162-72.

29. Spittle AJ, Treyvaud K, Doyle LW, et al. Early emergence of behavior and social-emotional problems in very preterm infants. J Am Acad Child Adolesc Psychiatry 2009;48:909-18.

30. Malone IB, Leung KK, Clegg S, et al. Accurate automatic estimation of total intracranial volume: a nuisance variable with less nuisance. Neuroimage 2015;104:366-72.

31. Adduru VR, Michael AM, Helguera M, et al. Leveraging Clinical Imaging Archives for Radiomics: Reliability of Automated Methods for Brain Volume Measurement. Radiology 2017;284:862-9.

32. Li X, Yang J, Gao J, et al. A robust post-processing workflow for datasets with motion artifacts in diffusion kurtosis imaging. PLOS One 2014;9:e94592.

33. Li X, Gao J, Wang M, et al. Rapid and reliable tract-based spatial statistics pipeline for diffusion tensor imaging in the neonatal brain: Applications to the white matter development and lesions. Magn Reson Imaging 2016;34:1314-21.

34. Yeatman JD, Dougherty RF, Myall NJ, et al. Tract profiles of white matter properties: automating fiber-tract quantification. PLOS One 2012;7:e49790.

35. Huang H, Shu N, Mishra V, et al. Development of human brain structural networks through infancy and childhood. Cereb Cortex 2015;25:1389-404.

36. Zhu T, Liu X, Gaugh MD, et al. Evaluation of measurement uncertainties in human diffusion tensor imaging (DTI)-derived parameters and optimization of clinical DTI protocols with a wild bootstrap analysis. J Magn Reson Imaging 2009;29:422-35.

37. Fortin JP, Parker D, Tunç B, et al. Harmonization of multi-site diffusion tensor imaging data. Neuroimage 2017;161:149-70.

38. Johnson WE, Li C, Rabinovic A. Adjusting batch effects in microarray expression data using empirical Bayes methods. Biostatistics 2007;8:118-27.

39. Fortin JP, Cullen N, Sheline Yl, et al. Harmonization of cortical thickness measurements across scanners and sites. Neuroimage 2018;167:104-20.

40. Acharya UR, Hagiwara Y, Sudarshan VK, et al. Towards precision medicine: from quantitative imaging to radiomics. J Zhejiang Univ Sci B 2018;19:6-24.

41. Lambin P, Leijenaar RTH, Deist TM, et al. Radiomics: the bridge between medical imaging and personalized medicine. Nat Rev Clin Oncol 2017;14:749-62.

42. Sagiv SK, Nugent JK, Brazelton TB, et al. Prenatal organochlorine exposure and measures of behavior in infancy using the Neonatal Behavioral Assessment Scale (NBAS). Environ Health Perspect 2008;116:666-73.
43. Aydlett L. Neonatal Behavioral Assessment Scale (NBAS). In: GOLDSTEIN S, NAGLIERI JA, Encyclopedia of Child Behavior and Development. Boston, MA: Springer US, 2011:1004-5.

44. Nugent JK. The competent newborn and the neonatal behavioral assessment scale: T. Berry Brazelton's legacy. J Child Adolesc Psychiatr Nurs 2013;26:173-9.

45. Canals J, Hernández-Martínez C, Esparó G, et al. Neonatal Behavioral Assessment Scale as a predictor of cognitive development and IQ in full-term infants: a 6-year longitudinal study. Acta Paediatr 2011:100:1331-7.

46. Bao XL, Yu RJ, Li ZS. 20 -item neonatal behavioral neurological assessment used in predicting prognosis of asphyxiated newborn. Chin Med J 1993;106:211-5.

47. Li Y, Xu X, Wu K, et al. Monitoring of lead load and its effect on neonatal behavioral neurological assessment scores in Guiyu, an electronic waste recycling town in China. $J$ Environ Monit $2008 ; 10: 1233-8$.

48. Zhu X, Li H, Zhang C. Clinical effects of Ganglioside and fructose-1, 6-diphosphate on neonatal heart and brain injuries after Asphyxia. Pak J Med Sci 2017;33:1199-204.

49. Albers CA, Grieve AJ, Test review: Bayley N. Bayley scales of infant and toddler development-third edition. San Antonio, TX: Harcourt assessment. Journal of Psychoeducational Assessment 2006;2007:180-90

50. Ranjitkar S, Kvestad I, Strand TA, et al. Acceptability and Reliability of the Bayley Scales of Infant and Toddler Development-III Among Children in Bhaktapur, Nepal. Front Psychol 2018;9:1265.

51. Manandhar SR, Dulal S, Manandhar DS, et al. Acceptability and Reliability of the Bayley Scales of Infant Development III Cognitive and Motor Scales among Children in Makwanpur. J Nepal Health Res Counc 2016;14:47-50.

52. Janssen AJ, Nijhuis-van der Sanden MW, Akkermans RP, et al. A model to predict motor performance in preterm infants at 5 years. Early Hum Dev 2009;85:599-604.

53. Morris C, Bartlett D. Gross Motor Function Classification System: impact and utility. Dev Med Child Neurol 2004;46:60-5.

54. Wood E, Rosenbaum P. The gross motor function classification system for cerebral palsy: a study of reliability and stability over time. Dev Med Child Neurol 2000;42:292-6.

55. Palisano RJ, Cameron D, Rosenbaum PL, et al. Stability of the gross motor function classification system. Dev Med Child Neurol 2006;48:424-8.

56. Ramenghi LA, Fumagalli M, Righini A, et al. Magnetic resonance imaging assessment of brain maturation in preterm neonates with punctate white matter lesions. Neuroradiology 2007;49:161-7.

57. Niwa T, de Vries LS, Benders MJ, et al. Punctate white matter lesions in infants: new insights using susceptibility-weighted imaging. Neuroradiology 2011;53:669-79.

58. Wisnowski JL, Blüml S, Paquette L, et al. Altered glutamatergic metabolism associated with punctate white matter lesions in preterm infants. PLoS One 2013;8:e56880.

59. Cornette LG, Tanner SF, Ramenghi LA, et al. Magnetic resonance imaging of the infant brain: anatomical characteristics and clinical significance of punctate lesions. Arch Dis Child Fetal Neonatal Ed 2002;86:171F-7.

60. Sun C, Bai L, Yan H, et al. Harmonization for DTI measurements mapping across sites in multi-center MRI study [abstract]: ISMRM, 2017:1844.

61. Wang M, Li X, Jin C, et al. Harmonization for fractional anisotropy measurement at 3T in healthy subjects [abstract]. ISMRM 2017:2410.

62. Zhao L, Zhang T, Li X, et al. Harmonization for cortical thickness across sites in multi-center MRI study [abstract]. ISMRM 2017:1447.

63. Prastawa M, Gilmore JH, Lin W, et al. Automatic segmentation of MR images of the developing newborn brain. Med Image Anal 2005;9:457-66.

64. Makropoulos A, Robinson EC, Schuh A, et al. The developing human connectome project: a minimal processing pipeline for neonatal cortical surface reconstruction. Neuroimage 2018;173:88-112.

65. Cusack R, Wild C, Linke AC, et al. Optimizing stimulation and analysis protocols for neonatal fMRI. PLoS One 2015;10:e0120202. 